\title{
Revisão da Literatura
}

\section{Qualidade de vida e espiritualidade}

\section{Quality of life and spirituality}

\author{
Raquel Gehrke Panzini ${ }^{1}$, Neusa Sicca da Rocha², Denise Ruschel Bandeira ${ }^{3}$, \\ Marcelo Pio de Almeida Fleck ${ }^{4}$ \\ ${ }^{1}$ Psicóloga, doutoranda em Psiquiatria pela Universidade Federal do Rio Grande do Sul (UFRGS) e mestra em Psicologia pela UFRGS. \\ ${ }^{2}$ Psiquiatra, mestra e doutoranda em Psiquiatria pela UFRGS. \\ ${ }_{3}^{3}$ Psicóloga, doutora em Psicologia pela UFRGS e coordenadora do Programa de Pós-Graduação em Psicologia na UFRGS. \\ ${ }^{4}$ Psiquiatra, doutor em Medicina pela UFRGS e coordenador do Grupo WHOOOL no Brasil.
}

Tradução: Alexandre Augusto Macêdo Corrêa

\section{Resumo}

Contexto: A espiritualidade tem sido apontada como uma importante dimensão da qualidade de vida. Objetivo: Apresentar revisão de literatura sobre qualidade de vida e espiritualidade, sua associação e instrumentos de avaliação. Método: Busca do tema-título nas bases de dados PsycINFO e PubMed/Medline entre 1979 e 2005. Resultados: A qualidade de vida é um conceito recente, que engloba e transcende o conceito de saúde, sendo composto de vários domínios ou dimensões: física, psicológica, ambiental, entre outras. Considerada a medida que faltava na área da saúde, tem sido definida como a percepção do indivíduo de sua posição na vida no contexto da cultura e sistema de valores nos quais vive e em relação aos seus objetivos, expectativas, padrões e preocupações. Há indícios consistentes de associação entre qualidade de vida e espiritualidade/religiosidade, por meio de estudos com razoável rigor metodológico, utilizando diversas variáveis para avaliar espiritualidade (por exemplo: afiliação religiosa, oração e coping religioso/espiritual). Também existem vários instrumentos de qualidade de vida válidos e fidedignos que avaliam a dimensão espiritual/religiosa. Conclusões: Novos estudos são necessários, entretanto, especialmente no Brasil. Tais estudos proverão dados empíricos a serem utilizados no planejamento de intervenções em saúde espiritualmente embasados, visando a uma melhor qualidade de vida.

Panzini, R.G. et al. / Rev. Psiq. Clín. 34, supl 1; 105-115, 2007

Palavras-chave: Qualidade de vida, espiritualidade, religiosidade, instrumentos de avaliação.

\begin{abstract}
Background: Spirituality has been considered as an important quality of life dimension. Objectives: To present a literature review about quality of life and spirituality, their association and measurement. Methods: Search for title words in PsycINFO and PubMed/Medline database between 1979-2005. Results: Quality of life is a new concept, that embraces and transcends the concept of health. It is composed by several domains or dimensions: physical, psychological, environmental, among others. Called "the missing measure in health", it has been defined as the individual's perception of his/her position in life, considering cultural and value systems in which he/she lives, and in relation to his/her goals, expectations, standards and worries. There are consistent evidences of the association between quality of life and spirituality/religiosity, obtained from studies with reasonable methodological rigor, using many variables to assess spirituality (for example: religious affiliation, prayer, and spiritual/religious coping). There are also several valid and reliable quality of life instruments that assess the spiritual/religious dimension. Conclusions: New studies are needed especially in Brazil. These studies will provide empirical data to be used to plan spiritually based health care interventions, aimingat a a better quality of life.
\end{abstract}

Panzini, R.G. et al. / Rev. Psiq. Clín. 34, supl 1; 105-115, 2007

Key-words: Quality of life, spirituality, religiosity, assessment. 


\section{Introdução}

Existe abundância de dados sobre o impacto da religião na vida das pessoas (Levin e Vanderpool, 1991). Apesar disso, enquanto a medicina oriental busca integrar de forma explícita a dimensão religiosa/espiritual ao binômio saúde-doença (Fabrega, 2000), a medicina ocidental como um todo, especialmente a psiquiatria, vinha tendo duas posturas principais em relação ao tema: negligência, por considerar esses assuntos sem importância ou fora da área de interesse principal; ou oposição, ao caracterizar as experiências religiosas dos pacientes como evidência de psicopatologias diversas (Sims, 1994). Historicamente ignorada por muitos psicólogos, a religião foi denominada por Larson e Larson o fator esquecido na saúde física e mental (Pargament et al., 1992). Nas últimas décadas, entretanto, este panorama tem se modificado em função do que Saad et al. (2001) nomearam espiritualidade baseada em evidências: atualmente existem centenas de artigos científicos mostrando uma associação entre espiritualidade/religião e saúde que é estatisticamente válida e possivelmente causal (Levin, 1994). Assim, os profissionais da saúde possuem indicações científicas do benefício da exploração da espiritualidade na programação terapêutica de virtualmente qualquer doença. A parede entre medicina e espiritualidade está ruindo: médicos e demais profissionais de saúde têm descoberto a importância da prece, da espiritualidade e da participação religiosa na melhora da saúde física e mental, bem como para responder a situações estressantes de vida (Epperly, 2000).

Mais recentemente, vem havendo uma preocupação com o estabelecimento de parâmetros mais amplos de avaliação de saúde que não apenas a morbimortalidade. Neste contexto, o interesse no desenvolvimento de medidas de desfecho como "bem-estar" e "qualidade de vida” tem tido notável crescimento (Fleck, no prelo), e indícios de associação com espiritualidade/religiosidade têm sido encontradas.

Neste artigo, serão revisados os conceitos e as possíveis relações entre espiritualidade/religiosidade e qualidade de vida, e sua aplicação na área da saúde, por meio da revisão de estudos científicos no tema-título obtidos das bases de dados PsycINFO e PubMed/Medline datados entre 1979 e 2005. Os artigos foram selecionados segundo a sua relevância científica e o objetivo de fornecer amplo panorama sobre qualidade de vida e espiritualidade em termos de conceitos, pesquisas e instrumentos.

\section{Conceitos religiosos/espirituais}

Os conceitos religiosos/espirituais não são consensuais. O Dicionário Oxford (Simpson e Weiner, 1989) define espírito como a parte imaterial, intelectual ou moral do homem. O termo espiritualidade envolve questões quanto ao significado da vida e à razão de viver, não limitado a tipos de crenças ou práticas. A religião é a "crença na existência de um poder sobrenatural, criador e controlador do Universo, que deu ao homem uma natureza espiritual que continua a existir depois da morte de seu corpo". Religiosidade é a extensão na qual um indivíduo acredita, segue e pratica uma religião. Embora haja sobreposição entre espiritualidade e religiosidade, a última difere-se pela clara sugestão de um sistema de adoração/doutrina específica partilhada com um grupo. Crenças pessoais podem ser quaisquer crenças/valores sustentados por um indivíduo e que caracterizam seu estilo de vida e comportamento. Pode haver sobreposição com espiritualidade, pois crenças pessoais não necessariamente são de natureza não-material, como o ateísmo. Complementando, Koenig et al. (2001) salientam a relação dos termos com a busca do sagrado, definindo religião como um sistema organizado de crenças, práticas, rituais e símbolos delineados para facilitar a proximidade com o sagrado e o transcendente (Deus, Poder Maior ou Verdade/Realidade Final/Máxima) e espiritualidade como a busca pessoal por respostas compreensíveis para questões existenciais sobre a vida, seu significado e a relação com o sagrado ou transcendente que podem (ou não) levar a ou resultar do desenvolvimento de rituais religiosos e formação de uma comunidade.

\section{Conceito de qualidade de vida}

A introdução do conceito de qualidade de vida (QV) como medida de desfecho em saúde surgiu a partir da década de 1970, no contexto do progresso da medicina. Este trouxe um prolongamento na expectativa de vida, na medida em que doenças anteriormente letais (por exemplo, infecções) passaram a ser curáveis ou a ter, pelo menos, controle dos sintomas ou retardo no seu curso natural. Assim, esse prolongamento se dá à custa do convívio com formas abrandadas ou assintomáticas das doenças. Passou a ser de grande importância, então, dispor de maneiras de mensurar a forma como as pessoas vivem esses anos a mais. De fato, Fallowfield (1990) definiu a QV como "a medida que faltava na área da saúde".

A QV possui intersecções com vários conceitos eminentemente biológicos e funcionais, como status de saúde, status funcional e incapacidade/deficiência; sociais e psicológicos, como bem-estar, satisfação e felicidade; e de origem econômica baseada na teoria "preferência" (utility). Entretanto, seu aspecto mais genérico (saúde é apenas um de seus domínios) tem sido apontado como seu grande diferencial e sua particular importância (Fleck, no prelo).

Seis grandes vertentes convergiram para o desenvolvimento do conceito de $\mathrm{QV}$ : 1) os estudos de base epidemiológica sobre felicidade e bem-estar; 2) a busca de indicadores sociais; 3) a insuficiência das medidas objetivas de desfecho em saúde; 4) a "satisfação do 
cliente"; 5) o movimento de humanização da medicina; e, 6) a psicologia positiva. Esta última insere-se na atual tendência para o desenvolvimento da pesquisa dos aspectos positivos da experiência humana, e a pesquisa em QV está em sintonia com a busca de estudar variáveis positivas da vida humana (Seligman e Csikszentmihalyi, 2000). Apoiado pelo conceito amplo da Organização Mundial da Saúde (OMS, 1946) de que saúde é "um estado de completo bem-estar físico, mental e social e não apenas a ausência de doença ou enfermidade”, o foco exclusivo na doença, que sempre dominou a pesquisa na área da saúde, vem cedendo espaço ao estudo das características adaptativas, como resiliência, esperança, sabedoria, criatividade, coragem e espiritualidade. Assim, podemos ver que religiosidade, espiritualidade e crenças pessoais não são temas alheios ao conceito de $\mathrm{QV}$, sendo, na verdade, uma de suas dimensões.

Ainda não há consenso definitivo na literatura sobre o conceito de qualidade de vida. Entretanto, é importante ressaltar a distinção entre os conceitos de padrão de vida e QV (Skevington, 2002). O primeiro compreende indicadores globais das características relevantes do modo de viver das sociedades e indivíduos, em termos socioeconômicos, demográficos e de cuidados básicos de saúde disponíveis. O segundo baseia-se em parâmetros que se referem à percepção subjetiva dos aspectos importantes da vida de uma pessoa, os quais podem ou não coincidir com indicadores de padrão de vida. O Grupo de Avaliação da Qualidade de Vida da Divisão de Saúde Mental da OMS (Grupo WHOQOL) propôs que essas percepções se originam na cultura à qual a pessoa pertence. Por isso, a questão cultural é fundamental na $\mathrm{QV}$, já que diferentes culturas tendem a priorizar diferentes aspectos. O Grupo WHOQOL pode ter sido o primeiro a incluir na definição de $\mathrm{QV}$ o componente cultural como parte integrante e fundamental, em vez de tratar sua influência como uma variável não relacionada. Esse grupo é uma colaboração entre pesquisadores, clínicos e cientistas que vêm trabalhando juntos há mais de 12 anos com base em protocolos internacionais consensuais, desenvolvidos de comum acordo a cada estágio de desenvolvimento do projeto (Skevington, 2002). A partir de uma perspectiva transcultural, esse grupo definiu qualidade de vida como "a percepção do indivíduo de sua posição na vida no contexto da cultura e sistema de valores nos quais ele vive e em relação aos seus objetivos, expectativas, padrões e preocupações" (WHOQOL Group, 1994) - uma das definições mais utilizadas atualmente.

\section{Grupos de pesquisadores em QV}

Além do Grupo WHOQOL, pesquisadores independentes e outros grupos de pesquisadores, subsidiados por diferentes instituições, também têm estudado QV por todo o mundo, como o IQOLA (International Society for Quality of Life Assessment) e o grupo ${ }^{1}$ de pesquisadores que construiu o SEIQOL (The Schedule for the Evaluation of Individual Quality of Life) (Beaton et al., 2000; Skevington, 2002). Esses grupos têm diferentes perspectivas quanto à universalidade ou relatividade do conceito de $\mathrm{QV}$, refletindo-se em abordagens objetivas ou subjetivas, respectivamente, na construção de instrumentos de avaliação da QV.

A visão universal foi apoiada pelos dados coletados pelo Grupo IQOLA, segundo o alto grau de similaridade encontrado entre os perfis do instrumento SF-36 provindos de quatro países europeus, e pelos dados coletados ao redor do mundo pelo Grupo WHOQOL, por meio dos instrumentos WHOQOL-100 e WHOQOL-bref. A visão relativa utiliza-se de uma abordagem ideográfica que procura entender o indivíduo como um ser único, considerando as muitas diferenças individuais entre as pessoas mais importantes que suas similaridades. Instrumentos como SEIQOL e SEIQOL-DW (McGee $e$ t al., 1991; Browne et al., 1997) acessam QV individualmente mediante entrevista semi-estruturada, em estudos com grupos particularizados, como indivíduos sofrendo de uma mesma doença (Waldron, 1999). Embora tenham demonstrado boa aceitabilidade e fidedignidade, são inapropriados para algumas populações, como idosos ou pacientes severamente doentes, por requerer um processamento de informação abstrato complexo (Povey, 2002). Já Bullinger (apud Skevington, 2002) salienta que dimensões que incluem tanto escalas genéricas quanto específicas de QV (domínios) demonstraram alto grau de correspondência entre diferentes instrumentos, permitindo revelar muitas diferenças entre culturas e entre indivíduos. Assim, a abordagem universal permitiria salientar tanto similaridades quanto diferenças no conceito de $\mathrm{QV}$ entre os povos.

Tal debate tem raízes na escolha e utilização de diferentes metodologias e técnicas de análise de dados, e sua questão-chave centra-se na possibilidade ou não de realizar medidas transculturais de QV (Skevington, 2002). A importância desse tipo de medida sobressai porque, por meio delas, é possível compartilhar resultados oriundos do mundo todo quanto aos tratamentos clínicos realizados e viabiliza a comparação de $\mathrm{QV}$ em diferentes culturas ou grupos sociais, permitindo verificar a universalidade ou não do conceito de $\mathrm{QV}$, contribuindo ao debate.

\section{Pesquisas relacionando QV e espiritualidade/religiosidade}

Vários estudos têm enfocado a relação entre QV e diversas variáveis religiosas/espirituais. Ferriss (2002)

1 C. A. O’Boyle, H. McGee, A. Hickey, C. R. B. Joyce, J. Browne, K. O’Malley e B. Hiltbrunner. 
examinou a relação entre religião e $\mathrm{QV}$ por meio de indicadores objetivos e subjetivos de QV. Encontrou a variável felicidade associada à freqüência/presença em serviços religiosos, a preferências proselitistas e a preferências doutrinárias, bem como a certas crenças relacionadas à religião, como a crença de que o mundo é bom ou mau, mas não à crença na imortalidade. $\mathrm{O}$ autor concluiu que: 1) organizações religiosas contribuem para a integração da comunidade, consequientemente aumentando a QV; 2) como a frequiência/presença em serviços religiosos esteve imperfeitamente associada com QV, outros fatores deveriam estar atuando; 3) a concepção americana de "vida boa" se baseia fortemente em ideais judaico-cristãos; 4) os princípios da religião podem atrair pessoas com disposição para a felicidade; 5) a religião pode explicar um propósito na vida que promova bem-estar. Indícios de associação positiva significativa entre QV e bem-estar espiritual foram encontrados em diferentes populações: em amostra multiétnica de 1.617 participantes (idade $\mu=54,6$ anos), quando do desenvolvimento e da validação do instrumento FACIT-Sp (The Functional Assessment of Chronic Illness TherapySpiritual Well-Being Scale) (Peterman et al., 2002) e em amostra de pacientes oncológicos, independentemente do tipo de neoplasia (Cella et al., 1999). Ainda, pacientes com variados tipos de neoplasias ginecológicas mostraram relação positiva entre QV e bem-estar espiritual, existencial e religioso (Gioiella et al., 1998).

Outro estudo confirmou que qualidade de vida em indivíduos HIV positivos ( $\mathrm{n}=40,80 \%$ homens; $25-54$ anos) estava diretamente relacionada a fé religiosa, afiliação religiosa e estado de saúde, que, com nível socioeconômico, contribuíam positiva e significativamente para os escores de QV dos participantes no Quality of Life Index (QLI). Variáveis independentes como idade, etnia e gênero não contribuíram para o modelo de regressão (Flannelly e Inouye, 2001). Em uma pesquisa com 44 viúvas de vítimas de incêndio (idade $\mu=37$ anos), a maioria das que se descreveu como mais religiosas relatou $\mathrm{QV}$ mais estável nos últimos cinco anos após a morte dos maridos. Frequiência religiosa, participação em eventos sociais e ser membro de alguma instituição religiosa também estiveram ligados à maior estabilidade na $\mathrm{QV}$ (Bahr e Harvey, 1979). Em um estudo com 560 pessoas aleatoriamente entrevistadas por telefone, para além da frequiência de oração, que não se revelou bom indicador, os aspectos qualitativos da reza e a forma de rezar foram as variáveis que apresentaram maior efeito sobre a QV (Paloma e Pendleton, 1989).

Uma variável que tem se mostrado associada à $\mathrm{QV}$ é o coping religioso/espiritual (CRE): uso da religião, espiritualidade ou fé para lidar com o estresse e os problemas de vida. Utilizando um índice global de QV (cinco itens graduados pelo observador/pesquisador em escala Likert de 3 pontos) (Spitzer et al., 1981), Pargament et al. (1998) verificaram que, em uma amostra que contou com 551 idosos hospitalizados gravemente doentes, 256 pessoas vítimas do ataque de bomba em Oklahoma e 540 universitários que haviam sofrido eventos de vida estressantes, maior utilização de CRE positivo não se correlacionou com QV nem depressão. No entanto, maior utilização do CRE negativo correlacionou-se moderadamente com níveis piores de QV e maiores de depressão. Os autores concluíram que a religião/espiritualidade pode ser fonte de alívio ou desconforto, de solução de problemas ou causa de estresse, dependendo de como a pessoa se relaciona com ela, ou seja, se utiliza estratégias de CRE positivo ou negativo. No acompanhamento de dois anos da amostra dos idosos hospitalizados deste estudo, com 268 respondentes (29,5\% mortos, $25,5 \%$ perda), o CRE foi preditor de resultados espirituais e mudanças na saúde mental/física. O CRE positivo esteve associado com melhoras na saúde e o CRE negativo foi preditor retrospectivo de declínio na saúde: desfechos (outcome) espirituais limitados, pior status funcional em atividades de vida diária, maior depressão e menor QV. Os autores concluíram que pacientes debatendo-se continuamente com questões religiosas podem estar particularmente em risco para problemas de saúde (Pargament et al., 2001), em função do uso de CRE negativo.

Utilizando a mesma medida de $\mathrm{QV}$ do estudo anterior, uma Brief RCOPE de 63 itens para medir CRE e uma forma abreviada de 22 itens da escala COPE (Carver $e t$ al., 1989) para avaliar coping não-religioso, Koenig et al. (1998) estudaram uma amostra com 577 pacientes acima de 55 anos. Com relação à saúde física, maior uso de coping, religioso ou não, esteve associado à pior saúde física. Essa associação foi mais forte e frequiente para o CRE negativo do que para o positivo, bem como para as estratégias de coping não-religioso menos saudáveis, como negação e comportamento desengajado. Aceitação foi o único coping não-religioso associado à melhor saúde física. Freqüiencia religiosa também esteve consistentemente associada à melhor saúde física. Em relação à saúde mental, estiveram associados a menor depressão e maior QV cinco estratégias de CRE positivo, frequiência religiosa e duas estratégias de coping não-religioso mais saudáveis/positivas: aceitação e receber suporte social/emocional. Associadas a maior depressão e pior QV esteve a maioria das estratégias de CRE negativo (8 de 9) e as estratégias de coping não-religioso menos saudáveis/negativas: comportamento desengajado, negação, uso de álcool e drogas. Todas as 12 estratégias de CRE positivo, freqüência religiosa, importância da religião e atividades religiosas privativas estiveram robustamente associadas a crescimento associado ao estresse, cooperatividade e crescimento espiritual. Estratégias de CRE negativo apresentaram associações menos frequientes, mais fracas, menos consistentes e ocasionalmente negativas com essas variáveis.

Estudo longitudinal sobre uso de instrumentos de QV para prever hospitalização e mortalidade em pacientes com doença pulmonar obstrutiva apontou que QV baixa seria um poderoso preditor de hospitalização e todas 
as causas de mortalidade, indicando que instrumentos auto-administráveis e breves poderiam identificar pacientes que poderiam beneficiar-se de intervenções preventivas (Fan et al., 2002). Se esses instrumentos fossem elaborados e/ou utilizados dentro de uma perspectiva transcultural, poderiam ainda viabilizar comparações entre diferentes grupos sociais, culturais ou diferentes condições/contextos de atendimento à saúde, informações importantes para políticas públicas no combate às epidemias (por exemplo, de HIV), em que diferentes grupos culturais necessitam de avaliação de QV no contexto de atendimento voluntário ou profissional, como testagem e aconselhamento (Skevington, 2002).

\section{Instrumentos de $\mathrm{QV}$ que avaliam aspectos espirituais/religiosos}

Vários estudos têm apontado a importância da religião/espiritualidade na qualidade de vida. Por exemplo, pacientes com tuberculose, antes que seus médicos, indicaram o aumento da espiritualidade como importante fator de QV resultante de sua doença e/ou tratamento (Hansel et al., 2004). Tais achados levaram aos instrumentos de QV genéricos (WHOQOL Group, 1998; Fleck et al., 1999), de QVRS (Peterman et al., 2002) e de QV relacionada a alguma doença específica (Zebrack e Chesler, 2001) a apresentarem/incluírem uma dimensão espiritual entre seus domínios.

Ross (1995) definiu a dimensão espiritual como dependendo de três componentes, revelados nas necessidades de encontrar significado, razão e preenchimento na vida; de ter esperança/vontade para viver; e de ter fé em si mesmo, nos outros ou em Deus. A primeira necessidade, apontada por Pargament (1997) como um dos objetivos-chave da religião e do CRE, é considerada uma condição essencial à vida, pois quando um indivíduo se sente incapaz de encontrar um significado, ele sofre em função de sentimentos de vazio e desespero (Ross, 1995). De fato, o sofrimento espiritual não identificado freqüentemente é o culpado em um plano terapêutico malsucedido na reabilitação de deficiências físicas ( $\mathrm{Da}-$ vis, 1994). Em um estudo com 10 mulheres com câncer e cinco homens com síndrome de imunodeficiência adquirida, aqueles que encontraram um significado para sua doença também apresentaram melhor QV. Eles definiram a dimensão espiritual como uma composição de três conceitos principais: crença em uma força superior, reconhecimento da mortalidade e atualização do eu (amor próprio + aceitação + encontrar significado na vida/na doença) (Fryback e Reinert, 1999).

A importância da dimensão espiritual/religiosa na $\mathrm{QV}$ e nas medidas de QV foi destacada por Robbins et al. (2001). Em estudo longitudinal com 60 pacientes com es- clerose lateral amiotrófica (ELA), examinaram a relação entre função física, QV e espiritualidade/religiosidade, e sua variação no tempo (linha base, três e seis meses). Escalas específicas de QV e passagem de tempo foram fatores preditores da QV dos pacientes. Apesar do progressivo declínio na função física, os escores gerais de QV e religiosidade pouco mudaram. Em contraste, o escore de QVRS específico para ELA diminuiu paralelamente ao declínio nos escores de função física, indicando que este seria primariamente uma medida dessa função. Os autores concluíram que a $\mathrm{QV}$ em pacientes com ELA parece ser independente da função física e instrumentos de QV que incluem a avaliação de fatores espirituais, religiosos e psicológicos produzem resultados diferentes em relação àqueles obtidos usando somente medidas do funcionamento físico.

À mesma conclusão sobre a importância da dimensão religiosa/espiritual na QV chegou o Grupo WHOQOL, quando organizou grupos focais por todo o mundo em 1991 e os participantes afirmaram que espiritualidade/ religião/crenças pessoais eram variáveis importantes em sua QV e saúde (Skevington, 2002). Embora, desde a Assembléia Mundial de Saúde de 1983, a inclusão de uma dimensão não-material ou espiritual de saúde venha sendo discutida extensamente a ponto de haver uma proposta para modificar o conceito clássico de saúde da OMS para "um estado dinâmico completo de bemestar físico, mental, espiritual e social e não meramente a ausência de doença” (WHO/MAS/MHP/98.2, 1998), essas variáveis não faziam parte da estrutura original do instrumento. Foram então incluídas quatro questões ${ }^{2}$, compondo o domínio espiritualidade, religiosidade e crenças pessoais (SRPB), que se uniu aos cinco já existentes (físico, psicológico, relações pessoais, meio ambiente e nível de independência), completando o WHOQOL-100 (100 itens, 24 facetas, 6 domínios). Mesmo assim, o domínio SRPB ainda era limitado, tendo se mostrado insuficiente nos testes de campo realizados em vários centros para cobrir uma área tão abrangente $\mathrm{e}$ complexa, mas, por razões pragmáticas, não foi possível desenvolvê-las mais nesse instrumento (Skevington, 2002). Salienta-se que o WHOQOL-bref, a versão abreviada desse instrumento, não contém os domínios nível de independência nem espiritualidade, mas contém um item de cada faceta componente destes alocados nos domínios físico e psicológico, respectivamente.

Para cobrir tal lacuna, o Grupo WHOQOL desenvolveu transculturalmente o Módulo Espiritualidade, Religiosidade e Crenças Pessoais (WHOQOL-SRPB), uma expansão do domínio SRPB do WHOQOL-100 (WHOQOL SRPB Group, 2005; Fleck et al., 2003). Esse instrumento vem sendo desenvolvido de forma colaborativa entre diversos centros da OMS, selecionados

2 Suas crenças pessoais dão sentido à sua vida? 2) Em que medida você acha que sua vida tem sentido? 3) Em que medida suas crenças pessoais Ihe dão força para enfrentar dificuldades? 4) Em que medida suas crenças pessoais Ihe ajudam a entender as dificuldades da vida? 
em países com diferentes níveis de industrialização e disponibilidade de serviços de saúde, por meio de uma série de etapas - sempre utilizando método de tradução interativo para garantir equivalência conceitual e semântica. Inicialmente, um grupo internacional de experts sugeriu facetas e itens relacionados à SRPB, os quais foram revisados por 92 grupos focais realizados em 15 países, em quatro continentes: Argentina, Brasil, Uruguai, Itália, Espanha, Lituânia, Turquia, Reino Unido, Egito, Israel, Índia, China, Japão, Tailândia e Malásia. Estes objetivavam revisar a importância e as definições das facetas e sugerir itens e/ou facetas para inclusão no questionário. Foram compostos por profissionais de saúde (independentemente de suas crenças); pessoas com doenças agudas, crônicas e terminais; pacientes que se recuperaram de doenças; ateus e membros da religião dominante em cada centro e de grupos religiosos minoritários.

Com base nos dados qualitativos e quantitativos dos níveis de importância, um total de 15 facetas representadas por 105 itens foi confirmado como relevante pelo Grupo WHOQOL-SRPB, em Genebra, e liberado para teste-piloto, aplicado em 18 centros $(n=5.087)$ [Argentina $(\mathrm{n}=221)$, Brasil (Porto Alegre, $\mathrm{n}=253$; Santa Maria, $n=253)$, Uruguai $(n=251)$, Itália $(n=376)$, Espanha $(n=$ 240), Lituânia $(\mathrm{n}=482)$, Turquia $(\mathrm{n}=240)$, Reino Unido $(\mathrm{n}=283)$, Egito $(\mathrm{n}=240)$, Quênia $(\mathrm{n}=480)$, Israel $(\mathrm{n}=$ 270), Índia (Bangalore, $n=240$; Pondicherry, $n=364$ ), China $(\mathrm{n}=259)$, Japão $(\mathrm{n}=226)$, Tailândia $(\mathrm{n}=118)$ e Malásia $(n=240)$ ], com o WHOQOL-100. Análises fatoriais e de correlação interitens, entre outras, foram conduzidas e o total de oito facetas foi selecionado ( $\mathrm{Co}$ nexão/Ligação a Ser ou Força Espiritual, Significado/ Sentido de Vida, Admiração, Totalidade e Integração, Força Espiritual, $\mathrm{Paz}$ Interior, Esperança e Otimismo, Fé), representadas por 32 itens, com resposta em escala Likert-5 pontos (Rocha et al., no prelo). Atualmente, o teste de campo para validação do WHOQOL-SRPB está sendo realizado em seis países: Uruguai, Espanha, Inglaterra, Israel, China e também no Brasil (Panzini, 2005). Os resultados desta última fase gerarão a versão final do instrumento.

Recentemente, comentando o artigo do WHOQOLSRPB Group (2005), Moreira-Almeida e Koenig (2006) levantaram a questão de que cinco fatores do WHOQOLSRPB (Significado/Sentido na Vida, Admiração, Totalidade e Integração, Paz Interior e Esperança e Otimismo) não seriam medidas de religião nem espiritualidade propriamente ditas, mas medidas de resultados ou consequiências de religiosidade. Em função disso, a WHOQOLSRPB e escalas como a FACIT-Sp e a Escala de Bem-Estar Espiritual, em que tal fato se repetiria, correriam o risco de estar sempre relacionadas à saúde mental por definirem espiritualidade por traços ou características humanas positivas. Além disso, assinalaram que mesmo as outras três facetas "com uma conexão mais direta à espiritualidade” (Conexão/Ligação a Ser ou Força Espiritual, Força
Espiritual e Fé) poderiam não refletir nenhum tipo de espiritualidade já que, pelas instruções, a pessoa poderia responder segundo um sistema pessoal de crenças que não fosse religioso nem espiritual. Como indício a favor de seu argumento, ressaltaram que as últimas três facetas foram justamente as que tiveram maiores escores entre os participantes que estavam doentes, representando o voltar-se à religião para o coping com a doença, e que o grupo focal de pacientes brasileiros sugeriu que fossem eliminadas questões sem clara relação com religiosidade - embora ateus e profissionais da saúde tenham criticado questões com conotação religiosa.

Revisando tal comentário, salientam-se alguns aspectos. Primeiro, o WHOQOL-SRPB é um instrumento de medida de qualidade de vida antes que de espiritualidade/religiosidade. Mede QV no que esta se relaciona com religião/espiritualidade e mede, também, QV relacionada a crenças pessoais, que podem ou não ser espirituais/religiosas. Assim, propicia que pessoas não-religiosas, nem espiritualizadas, respondam ao instrumento pelo fato de possuírem, em geral, um princípio ou filosofia de vida, muitas vezes baseado em código mora/ético ou na fé em algo (por exemplo, na ciência), que fortemente embasa/estrutura suas vidas - similarmente ao que pode fazer a religião/espiritualidade. Entende-se que a fé é "a confiança que se tem na realização de alguma coisa, a certeza de atingir determinado fim" (Kardec, 1996, p. 300), em suma, é acreditar, e isto pode manifestar-se no ser humano de diferentes formas. Segundo, o tema do fator Significado/Sentido de Vida é citado por Pargament (1997) como um dos objetivos-chave da religião, ao qual este dedica vários fatores de sua escala de coping religioso/espiritual, a RCOPE (Pargament et al., 2000), envolvendo, portanto, a relação com o sagrado e o transcendente - definição de espiritualidade citada por Moreira-Almeida e Koenig (2006). Terceiro, um indício de que os fatores do WHOQOL-SRPB medem algo além ou diferente do que apenas características humanas positivas está no fato de que, na análise fatorial conjunta com o WHOQOL-100 (WHOQOL SRPB Group, 2005), os fatores SRPB da dimensão espiritual carregaram em dimensão separada da dimensão psicológica, bem como de todas as outras dimensões daquele instrumento. Por fim, ressalta-se que o WHOQOL-SRPB é uma iniciativa pioneira por medir uma dimensão que não é rotineiramente avaliada em instrumentos de QV genérica, conforme se salientou no artigo original e no comentário.

Além do WHOQOL-100 e do WHOQOL-SRPB, outros instrumentos de QV também apresentam dimensões espirituais/religiosas. O Quality of Life-Cancer Survivors (QOL-CS) possui uma subescala de bem-estar espiritual, além das subescalas de bem-estar físico, psicológico e social que, com dois subcomponentes (medos e angústias), completam essa escala visual analógica de 41 itens. Suas propriedades psicométricas foram exploradas em amostra de 177 crianças sobreviventes de câncer, de 
16 a 29 anos (Zebrack e Chesler, 2001), tendo demonstrado boa consistência interna, validade concorrente e discriminante (grupos definidos por estado de saúde e nível social). Os autores concluíram que o instrumento mede domínios relevantes e distintos da $\mathrm{QV}$ para crianças sobreviventes de câncer, embora tenham sugerido que certas mudanças melhorariam a medida para essa população. Já o Self-Perception and Relationships Tool (S-PRT), uma medida subjetiva de QVRS validada em amostra clínica (pacientes psiquiátricos, cardiológicos, nefrológicos, oncológicos, com distúrbios do sono ou dor pélvica crônica), possui 36 itens dispostos em cinco fatores/domínios: Bem-estar Intrapessoal (físico, mental e emocional), Receptividade Interpessoal, Contribuição Interpessoal, Receptividade Transpessoal e Orientação Transpessoal. Os dois últimos são escalas/dimensões espirituais concernentes a características ou sentimentos ante a crenças e princípios universais ou à presença divina (Atkinson et al., 2004).

\section{Pesquisas brasileiras envolvendo QV, espiritualidade e CRE}

Embora a religiosidade seja um componente reconhecidamente importante na cultura brasileira, existem relativamente poucos estudos voltados para o seu impacto na qualidade de vida e suas relações com a saúde. A título de exemplo, serão descritas duas pesquisas investigando a relação entre QV e espiritualidade/religiosidade, realizadas no Rio Grande do Sul por atuais integrantes do grupo WHOQOL do Brasil (Panzini et al., no prelo). Artigos e resumos sobre essas pesquisas encontramse indexados nas bases de dados PsychINFO, Scielo e Bireme/BVS.

Em 2002, realizou-se um estudo transversal controlado (Rocha, 2002) com o objetivo de verificar a associação entre qualidade de vida, estado de saúde e níveis de espiritualidade, religiosidade e crenças pessoais (SRPB). Participaram da pesquisa 122 pacientes hospitalizados (54,1\% homens; idade, $\mu=45,15 \pm 15,54$; anos de estudo $=8,93 \pm 3,99 ; 46,7 \%$ casados; $73,1 \%$ católicos) e 119 indi- víduos saudáveis da comunidade (50,4\% homens; idade, $\mu=39,38 \pm 15,3 ;$ anos de estudo $=13,13 \pm 6,58 ; 44,5 \%$ casados; $71,4 \%$ católicos). Cada participante $(\mathrm{n}=241)$ respondeu aos instrumentos: Inventário de Depressão de Beck (BDI), Inventário de Desesperança de Beck (BHS), WHOQOL-100 e WHOQOL-SRPBi (Escala de importância dada às facetas do WHOQOL-SRPB, usada no teste-piloto). A amostra foi pareada por sexo, idade e religião. Testes $t$ de Student para amostras independentes demonstraram que os escores médios do BDI foram mais altos para os pacientes $(10,55 \pm 8,46)$, em comparação aos doentes $(5,54 \pm 5,68 ; \mathrm{p} \leq 0,0001)$. O mesmo ocorreu para os escores médios do BHS: $3,68 \pm 3,16$ e $2,76 \pm 2,65$ ( $1 \leq 0,007)$, respectivamente. Quanto ao WHOQOL-100, os pacientes demonstraram piores escores de $\mathrm{QV}$ do que os indivíduos saudáveis com diferença significativa em todos os domínios, exceto no domínio SRPB, no qual os pacientes apresentaram uma média um pouco maior, estatisticamente não-significativa (Tabela 1). Os dados são compatíveis com a literatura, que mostra maior uso da religião/espiritualidade em situações de enfrentamento (coping) de doenças (Pargament e Hahn, 1986; Siegel et al., 2001; Tix e Fraizer, 1998). Assim, a presença de uma doença pode estar associada à piora na maioria dos domínios de $\mathrm{QV}$, exceto no domínio SRPB.

Já a média dos pacientes no WHOQOL-SRPBi $(96,9)$ foi mais alta comparada à média dos saudáveis $(92,9$; $\mathrm{p} \leq 0,03)$. Após regressão múltipla, incluindo nível socioeconômico (NSE), essa diferença deixou de ser significativa, indicando que, apesar da pequena tendência de os indivíduos pacientes darem mais importância à dimensão espiritual/religiosa, os indivíduos saudáveis também a valorizaram bastante, considerando que a pontuação máxima é 100. Todavia, essa diferença poderia estar subestimada neste estudo, já que os indivíduos saudáveis eram membros praticantes de sua religião e, por isso, tenderiam a ter escores de religiosidade maiores que na população em geral. A diferença, portanto, poderia ser explicada pela maior necessidade de apoio que os pacientes apresentam para enfrentar as demandas associadas ao adoecimento

Tabela 1. Testes $t$ de Student para amostras independentes quanto ao estado de saúde, em relação às médias de qualidade de vida.

\begin{tabular}{l|c|c|c|c}
\hline Domínios do WHO00L-100 & $\begin{array}{c}\text { Paciente }(\mathbf{n}=\mathbf{1 2 2}) \\
\text { Média }(\mathbf{\pm} \mathbf{d} \mathbf{p})\end{array}$ & $\begin{array}{c}\text { Saudável }(\mathbf{n}=\mathbf{1 1 9}) \\
\text { Média }(\mathbf{\pm} \mathbf{d p})\end{array}$ & TEP & Magnitude de efeito \\
\hline Físico & $50,02(15,10)$ & $64,25(13,64)^{\mathrm{a}}$ & 1,0 & Moderado a grande \\
Psicológico & $62,72(12,25)$ & $69,10(11,82)^{\mathrm{a}}$ & 0,5 & Pequeno a moderado \\
Nível de independência & $55,76( \pm 19,43)$ & $80,24( \pm 11,25)^{\mathrm{a}}$ & 1,5 & Grande a muito grande \\
Relações sociais & $67,68( \pm 13,97)$ & $72,67( \pm 12,43)^{\mathrm{b}}$ & 0,4 & Pequeno a moderado \\
Ambiente & $56,91( \pm 10,90)$ & $63,48( \pm 10,42)^{\mathrm{a}}$ & 0,6 & Moderado \\
SRPB & $71,33( \pm 18,93)$ & $68,96(20,49)^{\mathrm{c}}$ & 0,1 & Trivial a pequeno \\
Geral & $68,18(12,92)$ & $79,29(12,04)^{\mathrm{a}}$ & 0,9 & Moderado a grande \\
\hline
\end{tabular}

${ }^{a} p \leq 0,0001 ;{ }^{b} p \leq 0,004 ;{ }^{c} p \leq 0,35 ;$ TEP: tamanho de efeito padronizado; $d p=$ desvio-padrão. 
(Koenig et al., 2001; Landis, 1996; Pargament e Brant, 1998), pela busca de um significado ou explicação para seu adoecimento (Ross, 1995) ou, até mesmo, pela tentativa de cura através da fé (Rabelo, 1993).

Também em um modelo de regressão múltipla, o WHOQOL-SRPBi apareceu positivamente associado aos domínios psicológico (beta $=0,17$ ), relações sociais (beta $=0,12)$, ambiente (beta $=0,11), \mathrm{SRPB}($ beta $=0,72) \mathrm{eQV}$ geral (beta $=0,10)(0,10 \leq p \leq 0,0001)$, quando ajustado para idade, NSE, sintomas depressivos (BDI) e estado de saúde. Isto demonstrou que a importância da espiritualidade/religiosidade/crenças pessoais pode estar positivamente associada com a maioria dos domínios de $\mathrm{QV}$. Tal achado corrobora a literatura, que mostra relação positiva entre religiosidade e relações sociais (Levin e Chatters, 1998a, 1998b; Levin e Vanderpool, 1987). Ainda, em relação ao domínio psicológico, sabe-se que a religiosidade pode estar associada a menores níveis de depressão (Braam et al., 1997; Koenig et al., 1998) e maiores índices de esperança e bem-estar (ElerhorstRyan, 1996), o que também pode explicar a correlação positiva com QV em geral.

Considerando os resultados, os autores concluíram: 1) que apesar de o aumento do WHOQOL-SRPBi (escores de importância das facetas SRPB) estar sofrendo influência de outros fatores, principalmente NSE, em relação à qualidade de vida, espiritualidade/religiosidade/crenças pessoais, teve uma associação positiva com alguns domínios de QV independentemente de outros fatores envolvidos (Rocha et al., 2002); 2) que o nível de evidência atual não permite recomendar aos pacientes que sejam mais religiosos ou se vinculem mais à dimensão não-material de forma tão categórica conforme já é estabelecida em relação a hábitos e comportamentos como beber e fumar, por exemplo; 3 ) que, embora se trate de um estudo transversal, não sendo possível estabelecer relações de causa e efeito, mas sim de associação, os dados apresentados sugerem que a dimensão não-material ou espiritual deve ser considerada como fator importante tanto no processo de adoecimento quanto no processo de coping com a doença.

Em 2004, em pesquisa transversal (Panzini, 2004) que validou a Escala de Coping Religioso-Espiritual (Escala CRE) (Panzini e Bandeira, 2005a), investigou- se a esperada relação entre qualidade de vida, coping religioso/espiritual (CRE) e saúde. Os 616 participantes (mulheres $=65 \%$; idade $\mu=41,38 \pm 18,44,13-82$ anos; solteiros $=46,8 \%$, casados $/$ vivendo como $=32,5 \%$; ensino superior $=52,1 \%$; renda até três salários mínimos $=$ $25 \%$, entre $5-10=24,1 \%$ ), acessados nos locais que freqüentavam [instituições religiosas e grupos espirituais não-institucionalizados $=74,4 \%$, universidades $=13,5 \%$, clínicas de tratamento de saúde $=9,1 \%$ e Internet (web mail $)=2,9 \%$, pertenciam a diversas religiões $/$ crenças: católicos $=40,4 \%$; espíritas $=31,5 \%$; espiritualizados sem religião $=8,3 \%$; evangélicos $=7,5 \%$; duas ou mais religiões simultâneas = 4,2\%; afro-brasileiros (africanistas ou umbandistas) $=3,9 \%$; outras religiões $=2,2 \%$ e ateus/agnósti$\cos =2 \%$. Além do Termo de Consentimento Esclarecido, foram utilizados os instrumentos WHOQOL-bref, Escala CRE e Questionário Geral.

Os resultados apontaram uma associação positiva geral entre CRE e QV (Tabela 2), já que os escores de CRE total, que consideram as médias dos escores de CRE positivo e CRE negativo, estiveram positivamente correlacionados com todos os domínios de QV do WHOQOL-bref. O índice CRE negativo esteve negativamente correlacionado à $\mathrm{QV}$ em níveis mais elevados do que o índice CRE positivo esteve positivamente correlacionado à $\mathrm{QV}$, indicando efeito prejudicial importante do CRE negativo na QV. Em função disso, propôs-se uma proporção mínima de uso de 2 CRE positivo : $1 \mathrm{CRE}$ negativo (valores de razão CRE negativo/CRE positivo $\leq 0,50)$ para que se obtenham resultados positivos do coping religioso/espiritual na qualidade de vida (Panzini e Bandeira, 2005b).

Testes $t$ de Student demonstraram que aqueles que possuíam maiores escores de qualidade de vida apresentaram maior uso de CRE positivo e menor de CRE negativo, indicando melhor utilização qualitativa do CRE. Esta foi a primeira confirmação empírica da proporção teórica proposta pela autora (Panzini, 2004), já que a razão CRE negativo/CRE positivo no grupo com $\mathrm{QV}$ alta $(\mathrm{n}=94)$ ficou abaixo do ponto de corte sugerido $(0,43)$ e a do grupo com QV baixa $(\mathrm{n}=95)$ ficou acima $(0,71 ; p \leq 0,0001)$. Novos testes demonstraram que os participantes que usaram mais coping religioso/espiritual apresentaram níveis maiores de $\mathrm{QV}$ em todos os

Tabela 2. Correlações Pearson ( $r$ ) entre qualidade de vida e coping religioso/espiritual ( $n=616)$.

\begin{tabular}{l|c|c|c|c|c}
\hline \multirow{2}{*}{ Escala CRE } & \multicolumn{4}{|c}{ WHOQOL-Bref } \\
\cline { 2 - 6 } & Físico & Psicológico & Relações sociais & Ambiente & Geral \\
\hline CRE total & 0,31 & 0,44 & 0,29 & 0,31 & 0,20 \\
CRE positivo & $0,11^{\mathrm{a}}$ & 0,21 & $0,12^{\mathrm{b}}$ & $0,08^{\mathrm{c}}$ & $0,06^{\mathrm{d}}$ \\
CRE negativo & $-0,34$ & $-0,38$ & $-0,28$ & $-0,37$ & $-0,24$ \\
Razão CRE negativo/CRE positivo & $-0,32$ & $-0,43$ & $-0,31$ & $-0,33$ & $-0,21$ \\
\hline
\end{tabular}

Estatisticamente significativos: ${ }^{a} p \leq 0,008 ;{ }^{b} p \leq 0,002$; demais $p \leq 0,0001$.

Estatisticamente não-significativos: ${ }^{c} p \leq 0,057 ;{ }^{d} p \leq 0,181$. 
domínios do WHOQOL-bref (Tabela 3), além de níveis mais altos de saúde objetiva, frequiência religiosa e crescimento espiritual, em comparação aos participantes que usaram menos. Por se tratar de estudo transversal, entretanto, não pôde ser estabelecida uma relação causal, ou seja, a direção da associação entre CRE e QV.

O estudo de Panzini (2004) corroborou dados de literatura (Koenig et al., 1998; Pargament et al., 1998; Pargament et al., 2001), demonstrando que também na população brasileira existe associação positiva entre coping religioso/espiritual e qualidade de vida e associação negativa entre CRE negativo e QV. O diferencial do estudo brasileiro, além da adaptação da Escala CRE, consiste a utilização de uma medida genérica, transcultural e abrangente de QV, o WHOQOL-bref. Esses resultados podem explicar parcialmente a ambigüidade dos dados relatados por diferentes pesquisas na relação espiritualidade/religiosidade com saúde e qualidade de vida, pois, enquanto existem inúmeras pesquisas recentes enfatizando o aspecto positivo, também pesa o cenário de outras, especialmente na área clínica das psicopatologias, lembrando a influência negativa que possam ter. Nesse sentido, a razão CRE negativo/CRE positivo pode ser um índice de avaliação importante, por oferecer a proporção entre as estratégias positivas e negativas de CRE utilizadas pelo avaliado, reforçando a importância de escalas abertas a ambos aspectos da religião/espiritualidade.

\section{Conclusões}

Apesar da falta de um consenso geral sobre os conceitos, a literatura científica tem demonstrado a existência de relação entre espiritualidade e qualidade de vida. Vários pesquisadores internacionais e nacionais, em grupo ou independentemente, têm se engajado em pesquisar esse tema, tendo desenvolvido instrumentos válidos e confiáveis para acessá-lo e produzido inúmeros estudos com razoável rigor metodológico. Ao finalizarmos, salientamos que o campo da qualidade de vida, mais recente, engloba e transcende o campo da saúde, envolvendo outras dimensões. No desenvolvimento dos estudos de QV, a importância e o envolvimento das questões espirituais cedo estiveram presentes. Portanto, entende-se que o campo da qualidade de vida pode vir a se tornar um mediador entre o campo da saúde e o das questões religiosas/espirituais, facilitando o desenvolvimento de intervenções em saúde espiritualmente embasadas, por pelo menos duas razões: por ser uma área de conhecimento mais recente e, portanto, com menos preconceito em relação à pesquisa em espiritualidade/religiosidade; e por o construto qualidade de vida ser mais amplo e multidimensional, exigindo o engajamento de profissionais de diferentes áreas do conhecimento para seu melhor entendimento, tornando-o transdisciplinar.

\section{Referências}

Atkinson, M.J.; Wishart, P.M.; Wasil, B.I.; Robinson, J.W. - The Self-Perception and Relations Tool (S-PRT): a novel approach to the measurement of objective health-related quality of life. Health and Quality of Life Outcomes 2: 36, 2004.

Bahr, H.M.; Harvey, C.D. - Widowhood and perceptions of change in quality of life: evidence from the Sunshine Mine Widows. Journal of Comparative Family Studies 10: 411-428, 1979.

Beaton, D.E.; Bombardier, C.; Guillemin, F.; Ferraz, M.B. - Guidelines for the process of cross-cultural adaptation of self-report measures. SPINE 24:3186-3191, 2000.

Braam, A.W.; Beekman, A.T.F.; Deeg, D.J.H.; Smit, J.H.; Van Tilburg, W. - Religiosity as a protective or prognostic factor of depression in later life: results from a community survey in The Netherlands. Acta Psychiatric Scand 96:199-205, 1997.

Browne J.P.; O’Boyle, C.A.; McGee, H.M.; McDonald, N.J.; Joyce, C.R.B. Development of a direct weighting procedure for quality of life domains. Quality of life Research 6, 301-309, 1997.

Carver, C.S.; Scheier, M.F.; Weintraub, J.K. - Assessing coping strategies: a theoretically based approach. J Pers Soc Psychol 56: 267-283, 1989.

Cella, D.; Brady, M.J.; Petterman, A.H.; Fitchett, G.A. - Case for including spirituality in quality of life measurement in oncology. Psycho-oncology 8: 417-428, 1999.

Davis, M.C. - The rehabilitation nurse's role in spiritual care. Rehabil Nurs 19(5):298-301, 1994.

Elerhorst-Ryan, J.M. - Instruments to measure spiritual status. In: Spilker B. Quality of life and pharmacoeconomics in clinical trials. 2.ed. LippincottRaven, Filadélfia, pp. 145-153, 1996.

Tabela 3. Médias* dos domínios de qualidade de vida do WHOQOL-bref para os grupos com alto e baixo escores de CRE total na escala CRE.

\begin{tabular}{l|c|c|c|c|c}
\hline \multirow{2}{*}{ Domínios do WH000L-bref } & CRE Total Alto $(\mathbf{n}=\mathbf{8 4})$ & CRE total вaixo $(\mathbf{n}=\mathbf{1 1 2})$ & & \\
\cline { 2 - 5 } & Média $( \pm \mathbf{d p})$ & Média $( \pm \mathbf{d p})$ & $\mathbf{t}$ & $\mathbf{p l} \leq$ \\
\hline Físico & $80,46( \pm 13,38)$ & $65,50( \pm 15,54)$ & $-7,145$ & 184,802 \\
Psicológico & $79,83( \pm 10,47)$ & $62,15( \pm 15,63)$ & $-8,884$ & 192 \\
Relações sociais & $76,96( \pm 15,06)$ & $60,49( \pm 21,22)$ & $-6,033$ & 193 \\
Ambiente & $70,93( \pm 13,99)$ & $56,67( \pm 15,57)$ & $-6,215$ & 184,039 \\
Geral & $78,62( \pm 13,32)$ & $69,60( \pm 18,36)$ & $-3,791$ & 192 & 0,001 \\
\hline
\end{tabular}

$d p=$ desvio-padrão.

*Médias ponderadas para valor máximo 100, diferentemente do estudo original (Panzini, 2004). 
Epperly, B.G. - Prayer, process, and the future of medicine. Journal of Religion and Health 39(1):23-37, 2000.

Fabrega, H. Culture, spirituality and Psychiatry. Current Opinion in Psychiatry 13:525-530, 2000.

Fallowfield L. - Quality of life: the missing measure in health care. Souvenir Press, New York, 1990.

Fan, V.S.; Curtis, J.R.; Tu, S.P.; McDonell, M.B.; Finn, S.D. - Using quality of life to predict hospitalization and mortality in patients with Obstructive Lung Diseases. CHEST 122: 429-436, 2002.

Ferriss, A.L. - Religion and the quality of life. Journal of Happiness Studies 3(3):199-215, 2002.

Flannelly, L.; Inouye, J. - Relationships of religion, health status and socioeconomic status to the quality of life of individuals who are HIV positive. Issues in Mental Health Nursing 22(3):253-272, 2001.

Fleck, M.P.A. - Problemas conceituais em qualidade de vida. In: Fleck, M.P.A. (org.) A avaliação da qualidade de vida em saúde: desenvolvimentos e aplicações do WHOQOL. Artes Médicas, Porto Alegre [no prelo].

Fleck, M.P.A.; Borges, Z.N.; Bolognesi, G.; Rocha, N.S. - Desenvolvimento do WHOQOL, módulo espiritualidade, religiosidade e crenças pessoais. Revista de Saúde Pública 37(4): 446-455, 2003.

Fleck, M.P.A.; Louzada, S.; Xavier, M.; Chachamovich E.; Vieira G.; Santos, L.; Pinzon, V. - Aplicação da versão em português do instrumento de avaliação de qualidade de vida da Organização Mundial da Saúde (WHOQOL-100). Rev Saúde Pública 33 (2): 198-205, 1999. Disponível em: www.fsp.usp.br/ rsp

Fryback, P.B.; Reinert, B. - Spirituality and people with potentially fatal diagnoses. Nursing Forum 34:13-22, 1999.

Gioiella, M.E.; Berkman, B.; Robinson, M. - Spirituality and quality of life in gynecologic oncology patients. Cancer Practice 6(6):333-338, 1998.

Hansel, N.N.; Wu, A.W.; Chang, B.; Diette, G.B. - Quality of life in tubercuIosis: patient and provider perspectives. Quality of Life Research 13: 639-52, 2004.

Kardec, A. - 0 evangelho segundo o espiritismo. Federação Espírita Brasileira, Rio de Janeiro, 1996 [1866]. 112.ed. Tradução de Guillon Ribeiro da 3.ed. francesa.

Koenig, H.G.; Larson, D.B.; Larson, S.S. - Religion and coping with serious medical illness. Ann Pharmacother 35:352-359, 2001.

Koenig, H.G.; Pargament, K.I.; Nielsen, J. - Religious coping and health status in medical ill hospitalized older adults. J Nerv Ment Dis 186(9):513-521, 1998.

Landis, B.J. - Uncertainty, spiritual well-being, and adjustment to chronic illness. Issues in Mental Health Nursing 17:217-231, 1996.

Levin, J.S. - Religion and health: is there an association, is it valid, and is it causal? Soc Sci Med 38(11):1475-482, 1994.

Levin, J.S.; Chatters, L.M. - Research on religion and mental health: an overview of empirical findings and theoretical issues. In: Koenig, H.G. (Org.). Handbook of Religion and Mental Health. Academic Press, London, pp. 33-50, 1998 .

Levin, J.S.; Chatters, L.M. - Religion, health, and psychological well-being in older adults. findings from national surveys. Journal of Aging and Health 10(4): 504-531, 1998b.

Levin, J.S.; Vanderpool, H.Y. - Is frequent religious attendance really conducive to better health?: Toward an epidemiology of religion. Social Science and Medicine 24(7):589-600, 1987.

Levin, J.S.; Vanderpool, H.Y. - Religious factors in physical health and the prevention of illness. Prevention in Human Services 9:41-64, 1991.

McGee, H.M.; O'Boyle, C.A.; Hickey, A.; O'Malley, K.; Joyce, C.R.B. - Assessing the quality of life of the individual: the $S E I O L L$ with a healthy and a gastroenterology unit population. Psychological Medicine 21:749-759, 1991.

Moreira-Almeida, A.; Koenig, H.G. - Retaining the meaning of the words religiousness and spirituality: a commentary on the WHOOOL SRPB group's "A cross-cultural study of spirituality, religion, and personal beliefs as components of quality of life" (62:6, 2005, 1486-97). Social Science and Medicine 63(4):843-5, 2006. [Errata em: Soc Sci Med, $63(10): 2753,2006$.]

Organização Mundial da Saúde. Constituição. OMS, New York, 1946.

Paloma, M.M.; Pendleton, B.F. - Exploring types of prayer and quality of life: a research note. Review of Religious Research 31:46-53, 1989.

Panzini, R.G. - Escala de Coping Religioso-Espiritual (Escala CRE):tradução, adaptação e validação da Escala RCOPE, abordando relações com saúde e qualidade de vida. (Dissertação de mestrado) Universidade Federal do Rio Grande do Sul, Porto Alegre, 238p., 2004. Disponível em: http://www.biblioteca.ufrgs.br/bibliotecadigital/

Panzini, R.G. - Aplicação da versão em português do WHOQOL - Módulo espiritualidade, religiosidade e crenças pessoais (SRPB) e investigação das relações entre saúde, qualidade de vida e SRPB. (Tese de doutorado). Faculdade de Medicina da Universidade Federal do Rio Grande do Sul, Porto Alegre, 40p., 2005.

Panzini, R.G.; Bandeira, D.R. - Escala de Coping Religioso-Espiritual (Escala CRE): elaboração e validação de construto. Psicologia em Estudo 10(3):507-16, 2005a.

Panzini, r.g.; Bandeira, D.R. - Quality of life and spiritual-religious coping relations. Qual Life Res 14(9):2106-2107, 2005b.

Panzini, R.G.; Rocha, N.S.; Bandeira, D.R.; Fleck, M.P.A. - Espiritualidade/ religiosidade e qualidade de vida. In: Fleck, M.P.A. (Org.) A avaliação da qualidade de vida em saúde: desenvolvimentos e aplicações do WH000L. Artes Médicas, Porto Alegre, no prelo.

Pargament, K.I. - The psychology of religion and coping: theory, research, practice. Guilford Press, New York, 548p., 1997.

Pargament K.I.; Brant C.R. - Religion and coping. In: Koenig, H.G. Handbook of Religion and Mental Health. Academic Press, London, pp. 111-128, 1998.

Pargament, K.I.; Hahn, J. - God and the just world: causal and coping attributions to God in health situations. Journal for the Scientific Study of Religion 25(2):193-207, 1986.

Pargament, K.I.; Koenig, H.G.; Perez, L.M. - The many methods of religious coping: Development and initial validation of the RCOPE. J Clin Psychol 56(4):519-543, 2000.

Pargament, K.I.; Koenig, H.G.; Tarakeshwar, N.; Hahn, J. - Religious struggle as predictor of mortality among medically ill elderly patients: a 2-year longitudinal study. Archival of International Medicine 13(27):1881-1885, 2001.

Pargament, K.I.; Smith, B.W.; Koenig, H.G.; Perez, L.M. - Patterns of positive and negative religious coping with major life stressors. Journal for the Scientific Study of Religion 37(4):710-724, 1998.

Pargament, K.I.; Olsen, H.; Reilly, B.; Falgout, K.; Ensing, D.S.; Haitsma, K.V. - God help me (II): the relationship of religious orientations to religious coping with negative life events. J Sci Study Relig 31(4):504-513, 1992.

Peterman, A.H.; Fitchett, G.; Brady, M.J.; Hernandez, L.; Cella, D. - Measuring spiritual well-being in people with cancer: the Functional Assessment of Chronic Illness Therapy-Spiritual Well-Being Scale (FACIT-Sp). Annals of Behavioral Medicine 24(1): 49-58, 2002.

Povey, R. - Quality of Life (4/9/2002) [On-line], 2002. Disponível em: http//:www. staffs.ac.uk/schools/sciences/psychology/coursematerials/healthypsychology/qualityoflife2002, acessado em 28/07/2004.

Rabelo, M.C. - Religião e cura: algumas reflexões sobre a experiência religiosa das classes populares urbanas. Cadernos de Saúde Pública 9(3):316-325, 1993.

Robbins, R.A.; Simmons, Z.; Bremer, B.A.; Walsh, S.M.; Fischer, S. - Quality of life in ALS is maintained as physical function declines. Neurology 56(4):442-444, 2001.

Rocha, N.S. - Associação entre estado de saúde, espiritualidade/religiosidade/crenças pessoais e qualidade de vida. (Dissertação de mestrado) Faculdade de Medicina da Universidade Federal do Rio Grande do Sul, Porto Alegre, 188p., 2002.

Rocha, N.S.; Borges, Z.N.; Fleck, M.P.A. - Health status and quality o life: the effect of spirituality/religiosity/personal beliefs. Qual Life Res 11(7):654, 2002

Rocha, N.S.; Panzini, R.G.; Silveira, J.; Fleck, M.P.A. - Desenvolvimento do módulo para avaliar espiritualidade, religiosidade e crenças pessoais do WHOQOL (WHOQOL-SRPB). In: Fleck, M.P.A. (Org.) A avaliação da qualidade de vida em saúde: desenvolvimentos e aplicações do WHOQOL. Artes Médicas, Porto Alegre, no prelo.

Ross, L. - The spiritual dimension: its importance to patients' health, wellbeing and quality of life and its implications for nursing practice. International Journal of Nurse Studies 32:457-468, 1995.

Saad, M.; Masiero, D.; Battistella, L.R. - Espiritualidade baseada em evidências. Acta Fisiátrica 8(3):107-112, 2001.

Seligman, M.E.; Csikszentmihalyi, M. - Positive psychology: an introduction. American Psychologist 55(1):5-14, 2000.

Siegel, K.; Anderman, S.J.; Schrimshaw, E.W. - Religion and coping with health-related stress. Psychology and Health 16(6):631-53, 2001. 
Simpson, J.A.; Weiner, E.S. - The Oxford English Dictionary. 2.ed. 20 V. Clarendon Press, Oxford, 1989.

Sims, A. - Psyche - Spirit as Well as Mind?. British Journal of Psychiatry, 165:441-46, 1994.

Skevington, S.M. - Advancing cross-cultural research on quality of life: observations drawn from the WHOQOL development. Quality of Life research 11:135-144, 2002.

Spitzer, W.0.; Dobson, A.J.; Hall, J.; Chesterman, E.; Levi, J.; Shepherd, R.; Battista, R.N.; Catchlove, B.R. - Measuring the quality of life of cancer patients. Journal of Chronic Diseases 34:585-597, 1981.

Tix, A.P.; Frazier, P.A. - The use of religious coping during stressful life events: main effects, moderation, and mediation. J Consult Clin Psychol 66(2):411-422, 1998.

Waldron, D.; O’Boyle, C. A.; Kearney, M; Moriarty, M.; Carney, D. - Qualityof-Life measurement in advanced cancer: Assessing the individual. Journal of Clinical Oncology, 17(11), 3603-3611, 1999.
WHO/MSA/MHP/98.2. - WHOOOL and Spirituality, Religiousness and Personal Beliefs (SRPB) - Report on WHO Consultation, pp.2-23, 1998.

WHOQOL Group. - The development of the World Health Organization quality of life assessment instrument (the WHOOOL). In: Orley, J. \& Kuyken, W. (Eds.) Quality of life assessment: international perspectives. Springer Verlag, Heidelberg, pp.41-60, 1994.

WHOQOL Group - The World Health Organization quality of life assessment (WHOQOL): development and general psychometric properties. Soc Sci Med 46:1569-1585, 1998.

WH000L SRPB Group. - A cross-cultural study of spirituality, religion, and personal beliefs as components of quality of life. Social Science and Medicine 62(6):1486-1497, 2005.

Zebrack, B.J.; Chesler, M.A. - A psychometric analysis of the Quality of LifeCancer Survivors (QOL-CS) in survivors of childhood cancer. Quality of Life Research: an International Journal of Quality of Life Aspects of Treatment, Care and Rehabilitation, 10(4):319-329, 2001. 